\title{
USB to USB and Mobile Data Transfer
}

\author{
R Ashwini ${ }^{1}$, Ramya ${ }^{2}$, Vanishree ${ }^{3}$, Uma B.B ${ }^{4}$ \\ Student, ECE, SMVITM, Udupi, India ${ }^{1,2,3,4}$
}

\begin{abstract}
Now day's portability is most important. So to achieve this, we are designing such a system which can carry anywhere. Using this system we can not only transfer the data but also we can see the transfer of the particular file which we want to send by using LCD display. Now a days to transfer a data between two pen drives we use PC or laptop, but it is not always possible to carry such a large device only for the data transfer. So to overcome this problem we design a system which is more compact. In our project we are transferring the data between two pen drives without using any computers or laptops. Whenever we insert two pen drives in to the USB port of Raspberry Pi, this can be done by giving the command to the processor. The processor indicates that the pen drive is inserted successfully till the user can not send any command to processor, the operation cannot start. After sending the particular command to processer, the processer will start fetching the data from source pen drive into buffer and the ARM processor wait for the signal from destination pen drive. When the processors get the signal from destination pen drive, so the data transfer operation begins. Only the ARM processor should get the external hard key input signal from the user
\end{abstract}

Keywords: RICS Machine, USB: - Universal Serial Bus, LED:-Light Emitting Diode, LCD:- Liquid Crystal Display.

\section{INTRODUCTION}

Several data and application are developed daily which common computer user has to transfer from one USB Flash device into another, with the minimum wastage of time.

The applications of the USB are computer peripherals such as keyboard, pointing devices, digital cameras, printers, portable media players, disk drives and network adapter, both to communicate and to supply electric power Several data and application are developed daily which common computer userhas to transfer from one USB Flash device into another, with the minimum wastage of time.

The applications of the USB are computer peripherals such as keyboard, pointing devices, digital cameras, printers, portable media players, disk drives and network adapter, both to communicate and to supply electric power. It has become common place on other devices, such as smart Phones, PDAs and video game consoles. USB has effectively replaced a variety of earlier interfaces, such as serial and parallel ports, as well as separate power chargers for portable devices. For this user has to first find a computer then wait for it to boot up, then plug in his device, and then transfer the data. Different types of USB devices are used now-a-days. It is not necessary that all of these devices are supported by the computer and the operating system and their device drivers are available and installed Carrying a computer or a laptop just for the sake of data transfer is not affordable these days in the age when people want all devices to be handy. Moreover, transferring data via a computer involves a lot of power to be wasted, since the computer has to be entirely functional before it can transfer data. Also, the threat of viruses and malware has made the life of computer users more complicated.

\section{II.LITERATURE SURVEY}

ARM 7 is used to implement USB to USB data transfer. ARM is important unit of the system because it reads data which we programmed and stored into its ROM, and then it executes it and controls the display as well as Vinculum VNC1L Embedded USB host controller. When key is press it read the data from source pen drive and writes to destination pen drive. Host controller recognizes the input peripheral as host or slaveaccording to firmware loaded in it. [1]

An electronic device capable of communicating locally with mobile devices through the USB interface is presented. The mobile devices will run a software application using the data sent by the developed module. The application will also manage the Internet connection to send that data to a remote central server. Because of this mobile devices can act in USB host or device mode, theproposed electronic gadget will is able to work both ways to expand the number of mobile devices which may be connected. The integration of external hardware and firmware with hardware and software for mobile devices leverages the core functionality of mobile devices for a large number of potential applications [2]. 


\section{IJIREEICE

ARM 7 is used to implement USB to USB data transfer. ARM is important unit of the system because it reads data which we programmed and stored into its ROM, and then it executes it and controls the display as well as Vinculum VNC1L Embedded USB host controller. When key is press it read the data from source pen drive and writes to destination pen drive. Host controller recognizes the input peripheral as host or slave according to firmware loaded in it [3].

An USB host in the embedded system is designed and realized; they selected the LPC2387 microprocessor to realize the design of USB host in the non-PC application situation. The LPC2387 microprocessor with one USB controller, by way of compiling program, implemented the detecting and recognition of USB disk and the data transmission from the host to the device as well as from the device to the host [4].

A module has been designed which can transfer data between pen drives and with any Bluetooth device. In the module to provide platform for USB a chip named as VDIP2 is used. This chip uses the VNC1L host controller to host the USB devices. VDIP2 is interfaced with ATMEGA32 microcontroller such that various task can be performed. VDIP2 provides the full access to VNC1L means all the ports and other pins directly accessible with the help of VDIP2. VNC1L operates in different modes and support many firmware. [5]

\section{METHODOLOGY}

The main objective of this project is to transfer data from one USB to other by using raspberry pi as main component.

\section{BLOCK DIAGRAM}

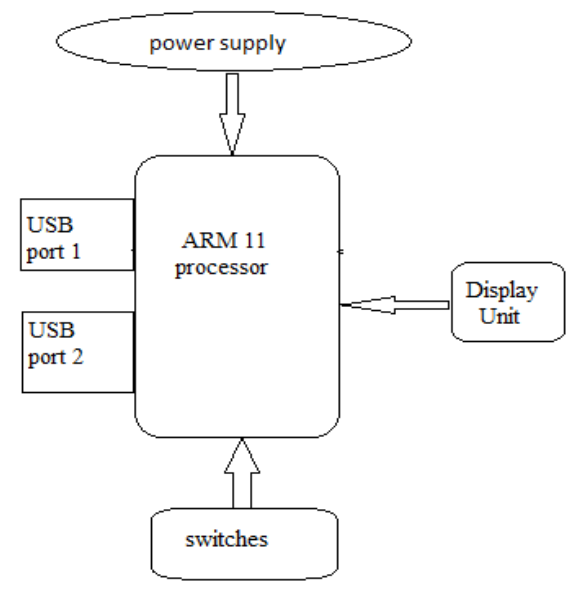

Fig 1 .Block diagram of proposed system

\section{HARDWARE DESCRIPTION RASPBERRY PI}

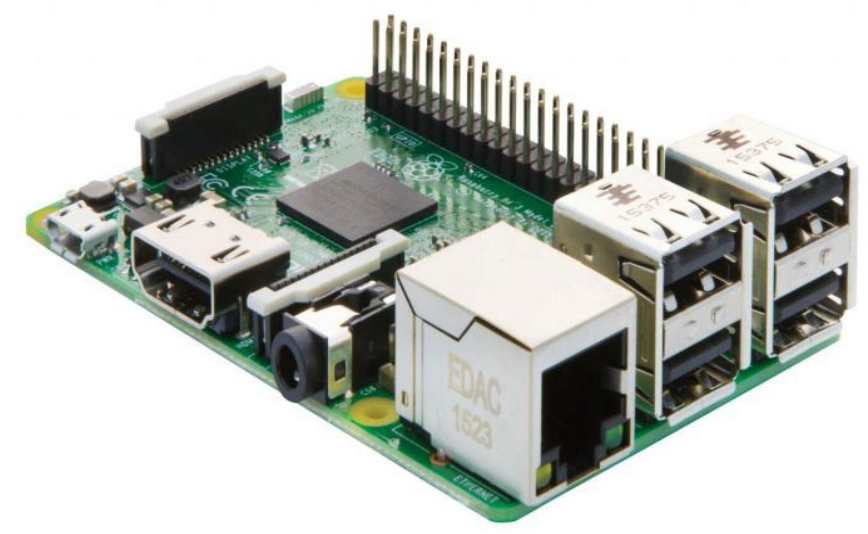




\section{ISO 3297:2007 Certified}

Vol. 5, Issue 5, May 2017

The hardware description of the project is as follows: The Raspberry Pi is a small computer about the size of a credit card. It was developed in the UK by the Raspberry Pi Foundation with the hope of inspiring a generation of learners to be creative and to discover how computers are programmed and how they function. The device uses the ARM processor which does most of the hard work in order to run the Raspberry Pi. The reason for this is that ARM processors are extremely efficient and fast when used in small devices. This makes the ARM processor the obvious choice for the Raspberry Pi.

Even though the Raspberry Pi is a computer it does not have a hard drive like traditional computers, instead it relies on the SD card for the starting up and storing of information. For the Raspberry Pi the SD card does the same job as a hard drive does in a traditional computer. The SD card must contain the operating system, programs and the data needed to run the Raspberry Pi. The operating system tells the Raspberry Pi how to function, how to handle any input from the user and how to manage programs when they are running.

Micro USB power port:-The micro USB power port is used to power the Raspberry Pi device.

HDMI Port:-The HDMI output is used to plug into a modern television or monitor.

Ethernet port:-The Ethernet port is used to connect the raspberry Pi to the internet or a local network.

USB port:-USB 2.0 ports are used to plug in a keyboard, mouse, external hubs etc.

Audio output:-The audio output can be used to plug into an external amplifier or an audio docking station.

GPIO headers:-The GPIO headers are used to connect the Raspberry Pi to other hardware device. For example, they can be used to connect to LED'S, motors and other electronics components.

RCA video output:-The video output is used to connect to an older type television.

ARM processor:-The ARM processor can be thought of as the brains of the Raspberry pi.

USB Ease of use was a major design goal for USB, and the result is an interface that's a pleasure to use for many reasons:

One interface for many devices:-USB is versatile enough for just about any standard PC peripheral function. Instead peripheral function, one interface serves many of having a different connector and cable type for each

Automatic configuration:-When a user connects a USB device to a PC, the operating system detects the device and loads the appropriate software driver. The first time the device connects, the operating system may prompt the user to insert a disc with driver software, but other than that, installation is automatic. Users don't need to reboot before using the device.

Easy to connect:-A typical PC has multiple USB ports, and hubs make it easy to add ports.

Wireless options:-USB originated as a wired interface, but technologies are now available for wireless communications with USB devices.

\section{WORKING}

The block diagram fig 1 gives a general idea to design our project. The over view of our project for designing purpose is given in the block diagram. The important hardware part which are used in the system are ARM 11 processor, Micro USB port gives $5 \mathrm{v}$ supply ,LCD display, four switches and USB interface. The main part of our system is the ARM processor; this is the heart of our project which is used for interfacing. The ARM processor provides the required functionality as per our requirement, so it is main intention to choice the ARM processor.

The USB connectors are connected to the ARM processor this two USB port are used to connect the other device such as keyboard, mouse, and external hubs. So as the Raspberry pi has the two USB port and it has the central host controller. The host controller manages attachment and removal of USB devices.

Here we use four switches, first switch for indication of mounted USBs, second switch for transferring data from USB1 to USB2, third switch for transferring data from USB2 to USB1, and last one for un mounting USBs.

When we pressed the specific switch it is sensed by the ARM processor as an interrupt, it provides the required operation as per we send the information, then the instructions is displayed on LCD. For LCD initialization and to make LCD in the working condition, the graphical LCD drives are installed on the ARM processor. LCD 16*2 display are connected which provide 20 characters. We have used the Linux OS for system which is more secured, open source and easily available for users. Linux kernel is ported in ARM's memory which manages device drivers and system libraries.

\section{FLOW CHART}

The fig 2 shows the working flowchart of the proposed module. When the module is initialized it will check for the insertion of pen drive. 


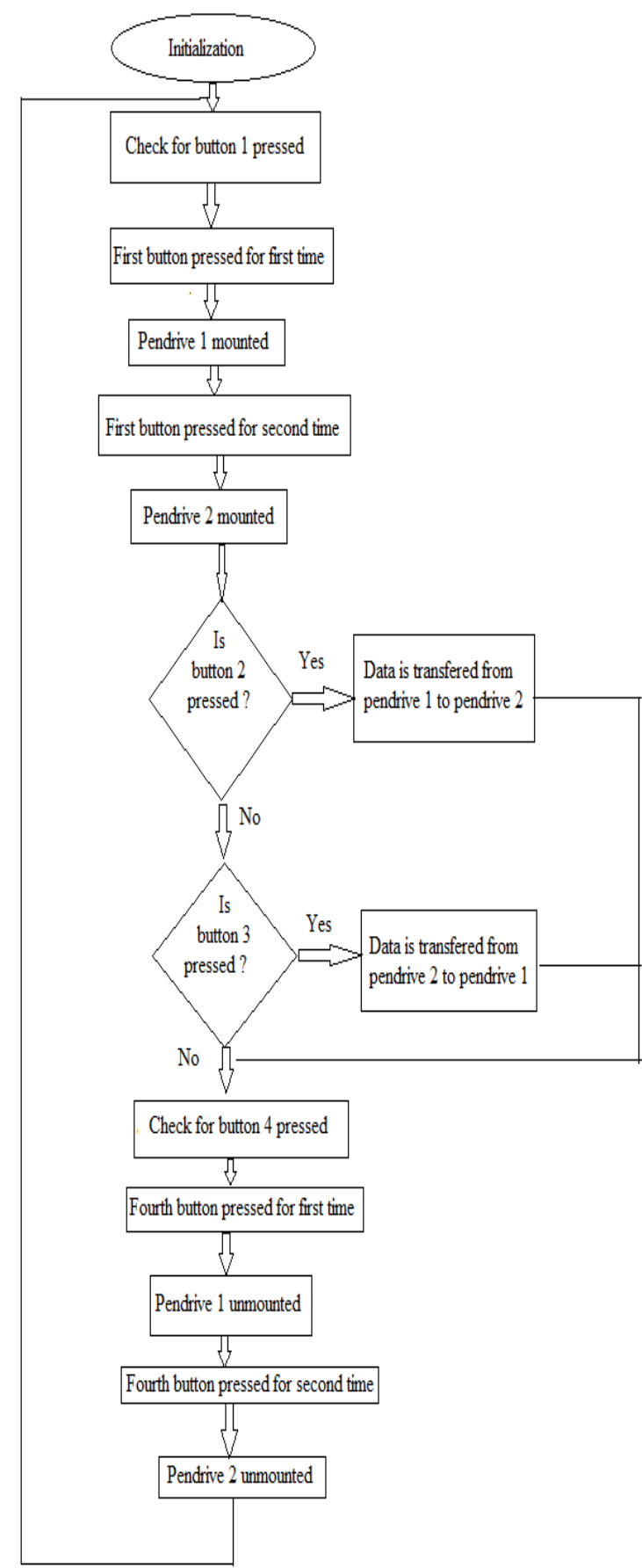

Fig 2.Flowchart of the proposed system

When first button is pressed for the first time, the pen drive 1 is mounted. When the same button is pressed for the second time, the pen drive 2 is mounted. It checks whether second button is pressed. If the second button is pressed then the data is transferred from pen drive 1 to pen derive 2. If second button is not pressed then it checks whether third button is pressed. When the third button is pressed the data from pen derive 2 to pen drive 3 is transferred. When fourth button is pressed for first time the pen drive 1 is unmounted. When the same button is pressed for second time the pen drive 2 is unmounted. Then it goes back to the initial condition.

\section{USB to Mobile Data Transfer:}

FTP stands, simply, for File Transfer Protocol. It's a basic way to host files in one location and access them from another. 
IJIREEICE

International Journal of Innovative Research in Electrical, Electronics, Instrumentation and Control Engineering

ISO 3297:2007 Certified

Vol. 5, Issue 5, May 2017

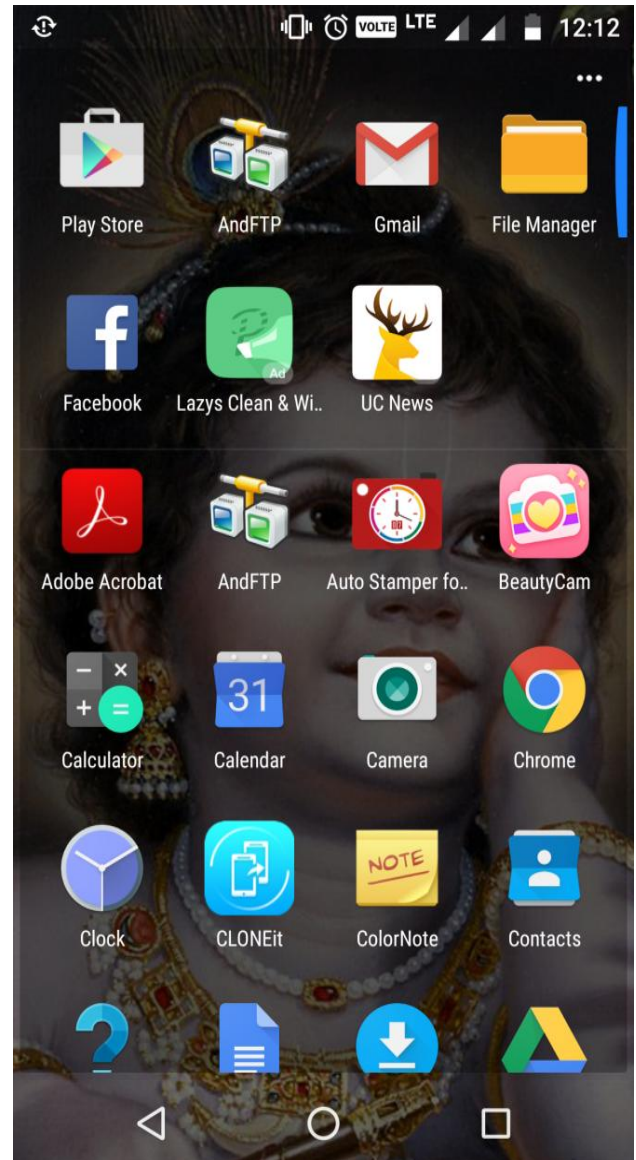

Fig 3.shows And FTP app in Android device

How to Use FTP to Move Files between USB and Android Device

In order to connect to your Android device, you need two things: an FTP server and an FTP client.

Here we are using AndFTP application for getting data from USB. AndFTP is a FTP/SFTP/SCP/FTPS client.It allows managing several FTP servers.

Once you have both the FTP client and server installed. It's time to set up the connection between the two. Let's start with the FTP client on the Android device. A network will be created between raspberry pi and mobile.

Once the networks are created then select the file that we have to download.

\section{RESULTS AND ANALYSIS}

A)USB to USB data transfer:

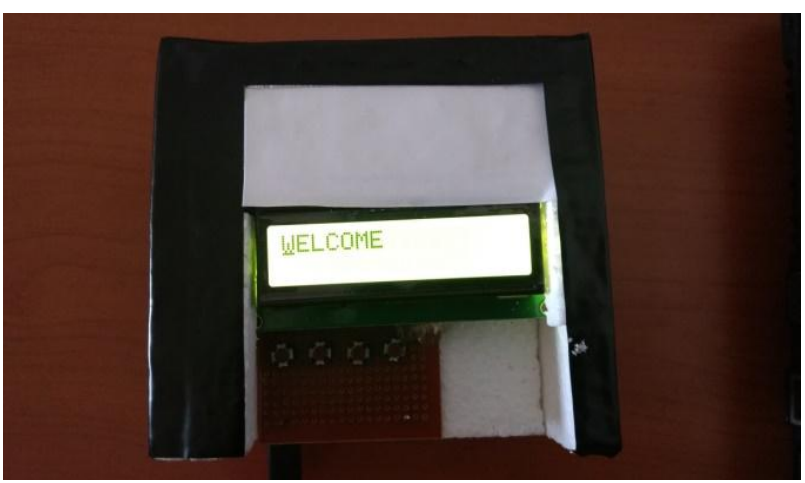

Fig 4.Initialization of the module 
IJIREEICE

International Journal of Innovative Research in Electrical, Electronics, Instrumentation and Control Engineering

ISO 3297:2007 Certified

Vol. 5, Issue 5, May 2017

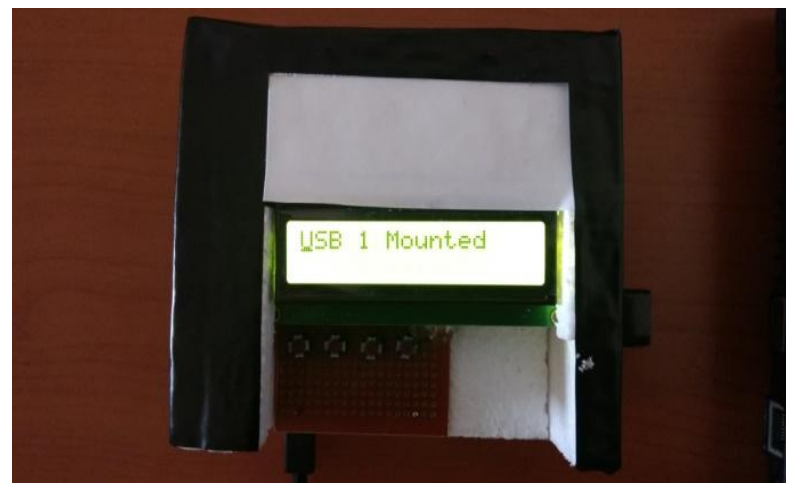

Fig 5.USB1 mounted

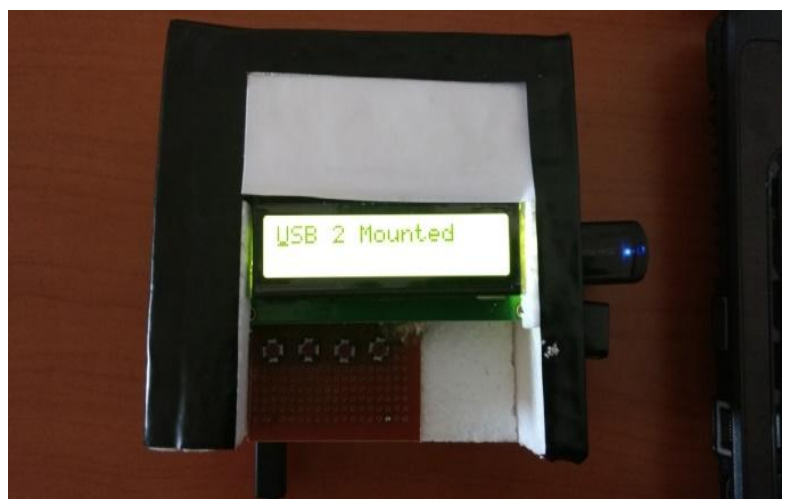

Fig 6.USB1 mounted

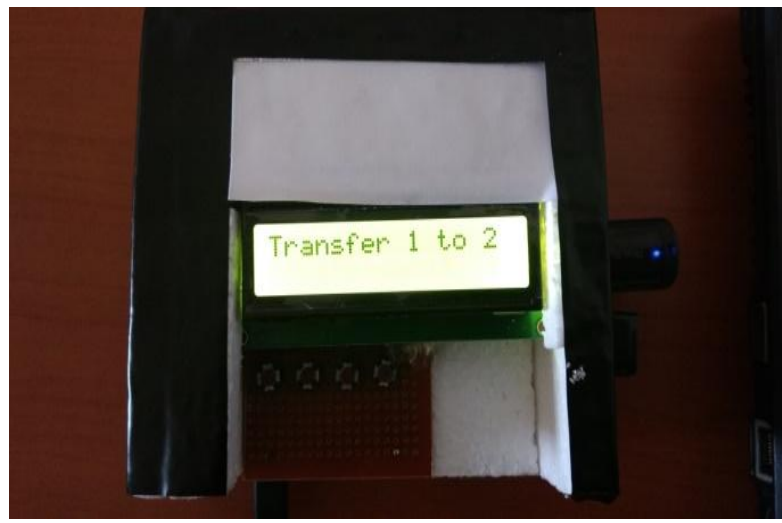

Fig 7.Transfer of data from USB1 to USB2

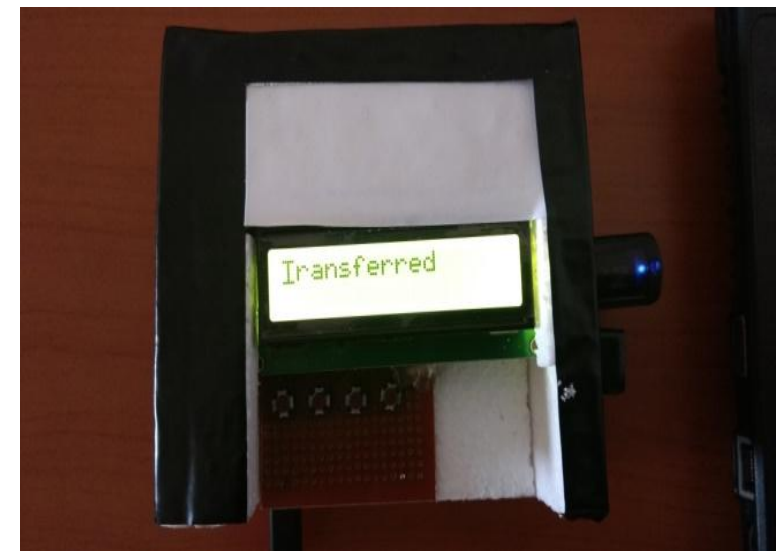

Fig 8.Completion of data transfer 
IJIREEICE

International Journal of Innovative Research in Electrical, Electronics, Instrumentation and Control Engineering

ISO 3297:2007 Certified

Vol. 5, Issue 5, May 2017

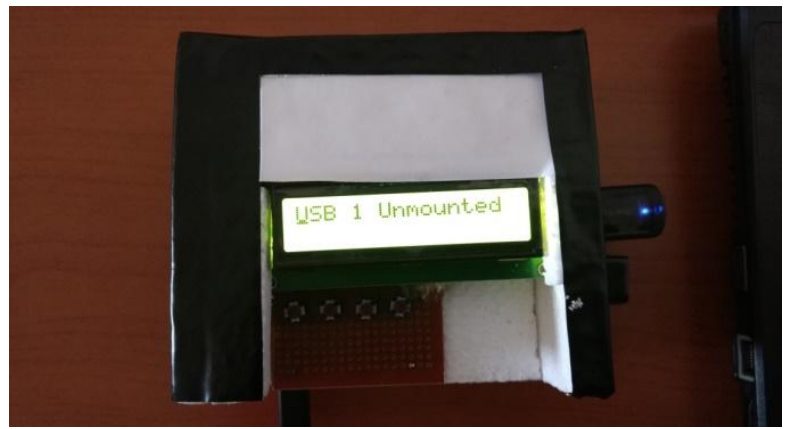

Fig 9. USB1 unmounted

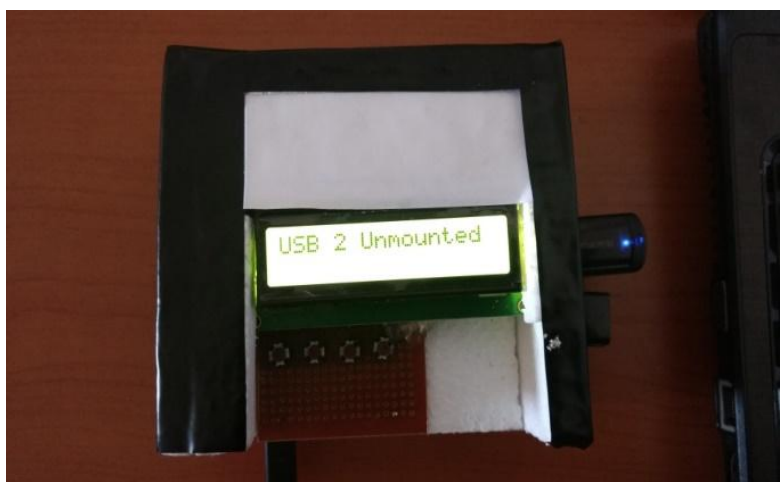

Fig 10.USB2 unmounted

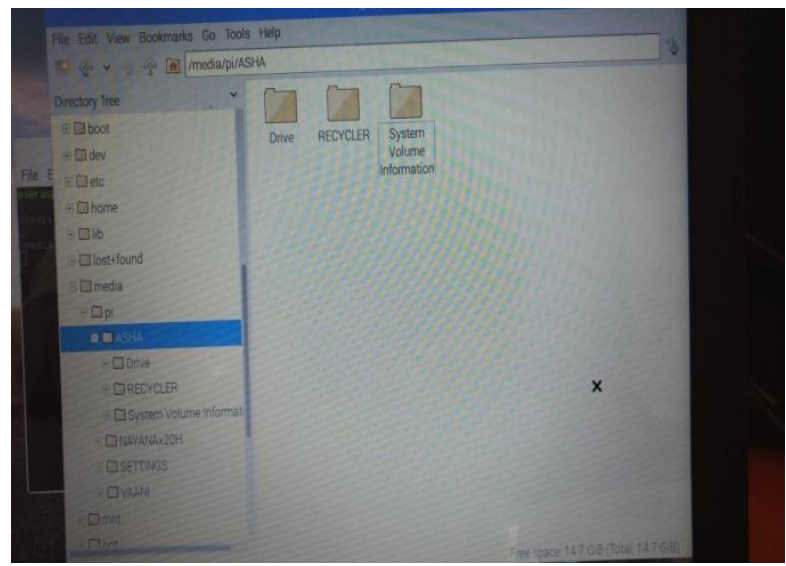

Fig 11.The folder of destination pen drive before and after data transfer

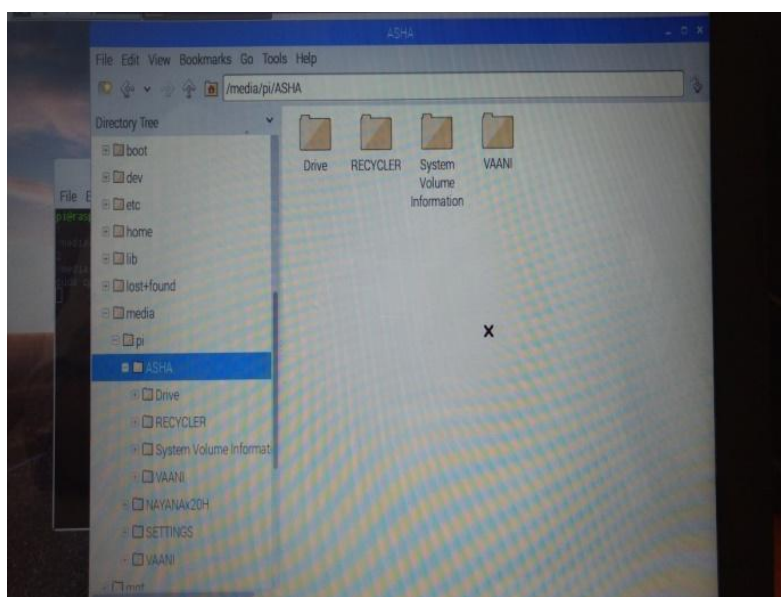

Fig 12.The folder of destination pen drive after data transfer 
International Journal of Innovative Research in Electrical, Electronics, Instrumentation and Control Engineering

ISO 3297:2007 Certified

Vol. 5, Issue 5, May 2017

B)Data transfer between mobile and USB

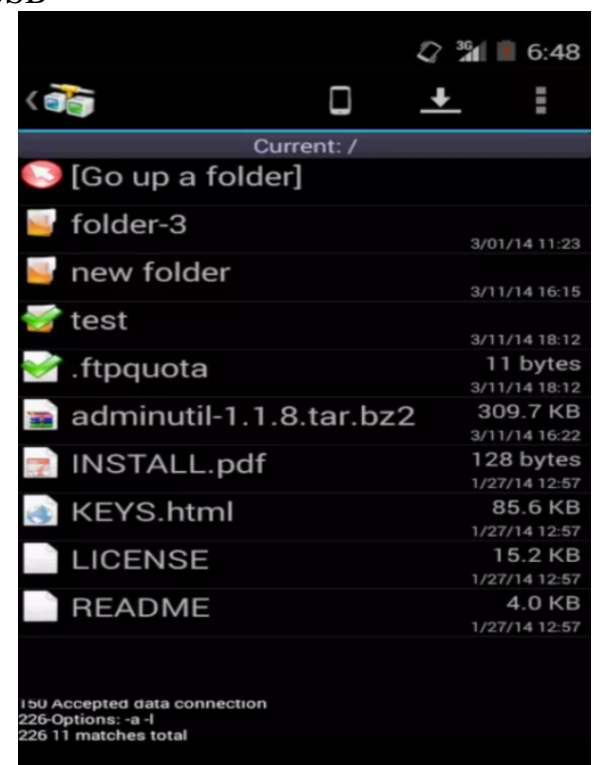

Fig 13.Folder shown before the data transfer in the mobile.

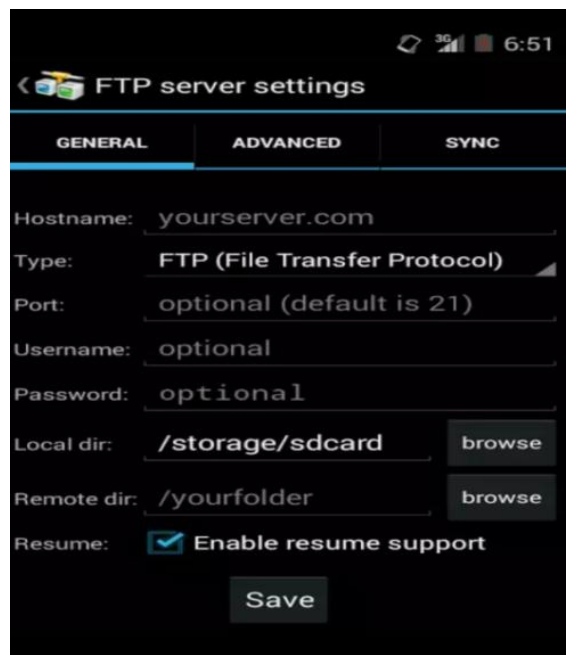

Fig 14. FTP server settings

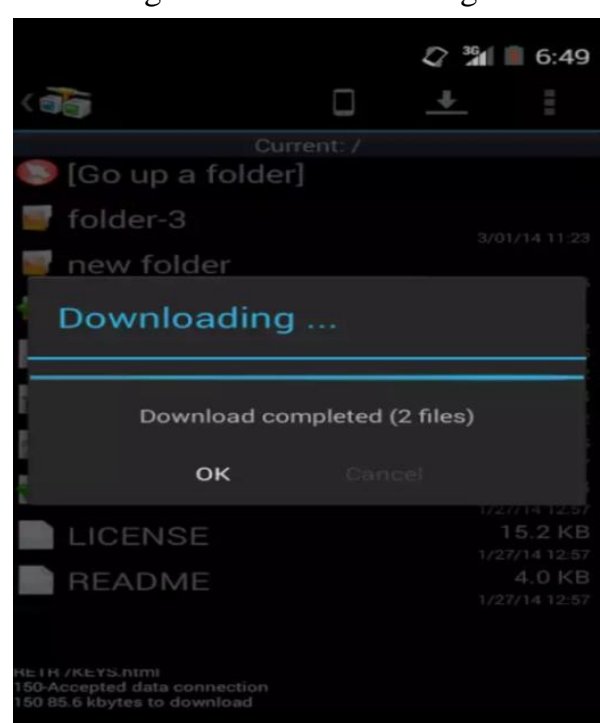

Fig 15.Shows downloading of files are completed 


\section{IJIREEICE \\ International Journal of Innovative Research in Electrical, Electronics, Instrumentation and Control Engineering \\ ISO 3297:2007 Certified \\ Vol. 5, Issue 5, May 2017}

\section{IV.CONCLUSION}

Transferring the data through USB in today's scenario is the most common task. But for transferring the data to a personal computer or laptop is difficult if one does not have any of them.It is affordable to purchase a USB data drive than purchasing a laptop or PC. Therefore this battery operated affordable device can transfer the data between two USB data drives and USB to mobile without the help of PC or laptop. The data transfer between USB to mobile is done by using AndFTP app.

\section{ACKNOWLEDGEMENT}

We are thankful to Management of Shri Madhwa Vadiraja Institute of Technology and Management and all our faculty members of E\&C Department for their encouragement and whole hearted cooperation. We would like to thank Prof.Aruna M.S for his guidance and valuable Suggestions time to time. We would like to express Profuse thanks to all for their support during this work.

\section{REFERENCES}

[1] USBFlashDrive.org [site], "USB Flash Drives, USB Memory and Portable Computer Hard Drive Information,"

[2] Ducloux J and Petrashin P. An Embedded USB dual role System Integrated for mobile devices. IEEE,2012.

[3] Gawali VS and Agarkar AM. Pen Drive to Pen Drive and Mobile Data Transfer Using ARM. IOSR Journal of Electronics and Communication Engineering.

[4] Xiaoyan Z and Yong T. Design and Realization of an embedded storage system based on LPC2387 microprocessor. IEEE, 2010

[5] Harpreet Singh1, Kamaldeep Kaur2. Design Module For Data Transfer Between Bluetooth Device And Flash Drive Using USB Host Controller. 